\title{
Replication-selective microbiological agents: fighting cancer with targeted germ warfare
}

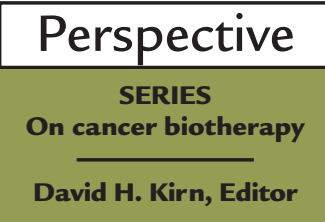

\author{
David H. Kirn \\ Imperial Cancer Research Fund, Molecular Oncology Unit, Program for Viral and Genetic Therapy of Cancer, \\ Imperial College School of Medicine, Hammersmith Hospital, Du Cane Road, London W12 ONN, United Kingdom. \\ Phone: 44-181-383-8584; Fax: 44-181-383-3258; E-mail: d.kirn@icrf.icnet.uk.
}

This Perspective series on cancer biotherapy will review the emerging field of tumor-targeted microorganisms. The clinical efficacy of any cancer treatment depends on both its antitumoral potency and the therapeutic index between cancerous cells and normal cells. Most current nonsurgical therapies for solid tumors fail on one or both scores, and it is unlikely that changes in dose or frequency, or even the combination of standard cytotoxic chemo- or radiotherapies, will satisfy both of these standards. Novel therapeutic approaches are required that offer greater potency and selectivity and that act by mechanisms not subject to cross-resistance when combined with standard therapies. Tumor-targeted microorganisms have the potential to fit all of these criteria. Their ability to replicate in tumor tissue allows for a massive amplification of the input "dose" (e.g., 1,000- to 10,000-fold increases) at the tumor site, while their lack of replication in normal tissues allows for efficient clearance and reduced toxicity.

Infectious organisms such as viruses and bacteria have evolved to replicate and spread in human tissues. In many cases, infection leads to tissue destruction, either through direct or immune-mediated effects (Table 1). Not long after the discovery and characterization of bacteria and viruses, investigators conceived that the cell-killing potential of these agents could be used to destroy cancer cells (1). Over the last century, diverse viruses - adenovirus, Bunyamwara, coxsackie,
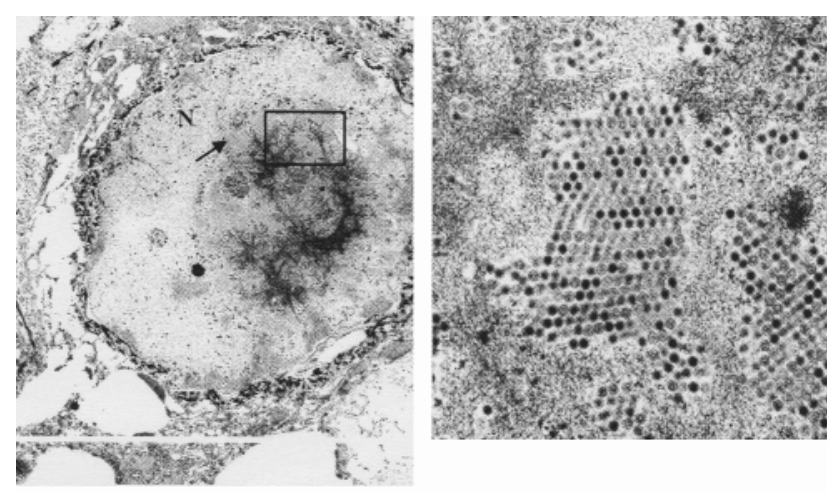

Figure 1

Electron micrograph of a squamous carcinoma cell from a patient following intratumoral injection with ONYX-015. This engineered strain of adenovirus replicates selectively in tumor cells and causes tumor-specific cytotoxicity. The higher magnification image, shown at the right, demonstrates that adenovirus particles form large arrays within the nucleus of infected tumor cells. dengue, feline panleukemia, Ilheus, mumps, Newcastle disease, vaccinia, and West Nile (2-4) - were injected into cancer patients by various routes, and bacterial agents have also been used as cancer therapies. Since the original description by Coley of tumor regressions following bacterial injection $(5,6)$, a variety of bacterial agents have been used as immune adjuvants, including Bacillus Calmette-Guérin (BCG) which eventually became the standard of care for superficial recurrent bladder carcinoma (7).

These studies illustrated both the promise of cancer biotherapy and the hurdles to be overcome. Both bacteria and viruses replicated within tumors and spread to distant tumor sites and many patients benefited from some degree of tumor necrosis, but patients were generally not properly evaluated by objective tumor response criteria. Many of the tumor responses appear to have been transient or incomplete, and while some of these agents were well-tolerated, toxicity occurred in some trials.

Since the time of these pioneering but poorly controlled clinical trials, revolutionary advances in molecular biology and genetics established mechanisms for carcinogenesis as well as for microbial replication and pathogenicity. These advances also allowed novel agents to be engineered to improve their safety and/or their antitumoral potency. In recent years, genetically engineered microorganisms in development have included herpesviruses (8) (R. Martuza, in this Perspective series), adenoviruses (9) (C. Heise and D. Kirn, this series), vaccinia (10) (M. Mastrangelo et al., this series) and Salmonella species (11) (M. Sznol et al., this series). In addition, the inherent tumor-selectivity of agents such as reovirus has been characterized at the genetic level (K. Norman and P. Lee, this series). Each of these agents has shown tumor selectivity in vitro and in vivo, and work in murine tumor models has established the efficacy of these treatments.

Until recently, detailed biological data from clinical trials was lacking, but now, as described by C. Heise and D. Kirn (this series), reports from ongoing clinical trials show that the engineered adenoviral mutant $d l 1520$ (ONYX-015, now known as CI-1042, Park-Davis Pharmaceuticals, Inc., division of Warner Lambert, Inc. Ann Arbor, Michigan, USA) replicates and induces necrosis selectively in human tumors (12). Neutralizing antibodies to the virus do not demonstrably prevent antitumoral activity following intratumoral injection. The adenovirus can be delivered to tumors through intra- 
Table 1

Replication-selective microbiological agents in clinical trials for cancer patients

\begin{tabular}{|c|c|c|c|}
\hline Parental strain & Agent & $\begin{array}{l}\text { Cell phenotype allowing } \\
\text { selective replication }\end{array}$ & Genetic alterations \\
\hline \multicolumn{4}{|l|}{ Engineered } \\
\hline Adenovirus ( $2 / 5$ chimera) & ONYX-015 & $\begin{array}{l}\text { Cells lacking p53 function } \\
\text { (e.g., deletion, mutation, HPV infection) }\end{array}$ & E1B-55kD, E3b gene deletion \\
\hline \multirow[t]{2}{*}{ Herpes simplex virus-1 } & G207 & Proliferating cells & $\begin{array}{l}\text { ribonucleotide reductase disruption } \\
\text { (lac- } Z \text { insertion into ICP6 gene) }\end{array}$ \\
\hline & & & $\begin{array}{l}\text { neuropathogenesis gene mutation } \\
\text { ( } \gamma 34.5 \text { gene) }\end{array}$ \\
\hline Adenovirus (serotype 5) & CN706 & Prostate cells (malignant, normal) & E1A expression driven by PSE element \\
\hline Adenovirus ( $2 / 5$ chimera) & Ad5-CD/tk-rep & $\begin{array}{c}\text { Cells lacking p53 function } \\
\text { (e.g., deletion, mutation, HPV infection) }\end{array}$ & $\begin{array}{l}\text { E1B-55kD gene deletion } \\
\text { Insertion of HSV-tk/CD fusion }\end{array}$ \\
\hline Vaccinia virus & $\begin{array}{l}\text { Wild-type +/- } \\
\text { GM-CSF }\end{array}$ & Unknown & $\begin{array}{c}\text { None for selectivity } \\
\text { Immunostimulatory gene (GM-CSF insertion) }\end{array}$ \\
\hline \multirow{2}{*}{\multicolumn{2}{|c|}{ (mechanism unknown:? nutrient, hypoxia, }} & Extracellular proliferation in tumor milieu & $\begin{array}{c}\text { Deletion of } m s b B \text { (lipidA metabolism) } \\
\text { auxotrophic mutations }\end{array}$ \\
\hline & & immune clearance differences) & \\
\hline \multicolumn{4}{|l|}{ Nonengineered } \\
\hline Newcastle disease virus & 73-T & Unknown & $\begin{array}{c}\text { Unknown } \\
\text { (serial passage on tumor cells) }\end{array}$ \\
\hline Autonomous parvoviruses & $\mathrm{H}-1$ & $\begin{array}{l}\text { Transformed cells } \\
\uparrow \text { proliferation } \\
\downarrow \text { differentiation } \\
\text { ras, p53 mutation }\end{array}$ & None \\
\hline Reovirus & Reolysin ${ }^{A}$ & $\begin{array}{c}\text { Ras-pathway activation } \\
\text { (e.g., ras mutation, EGFR signaling) }\end{array}$ & None \\
\hline
\end{tabular}

ANot yet in clinical trials; to enter clinical trials in 2000. HPV, human papillomavirus; PSE, prostate-specific enhancer; LPS, lipopolysaccharide; EGFR, epidermal growth factor receptor.

Table 2

Potential mechanisms of antitumoral efficacy with replication-selective microorganisms

\begin{tabular}{cc} 
Mechanism & $\begin{array}{c}\text { Examples }^{\mathrm{A}} \\
\text { Herpesvirus } \\
\text { I. Direct cytolysis during viral shedding } \\
\text { Reovirus } \\
\text { Vaccinia }\end{array}$ \\
II. Direct cytotoxicity due to viral proteins & $\begin{array}{c}\text { Adenovirus } \\
\text { Autonomous parvovirus }\end{array}$ \\
III. Augmentation of antitumoral immunity & \\
CTL infiltration, killing & Vaccinia \\
Tumor cell lysis, antigen release & Herpesvirus \\
Immunostimulatory cytokine induction & Adenovirus \\
Antitumoral cytokine induction (e.g., TNF) & Newcastle disease virus \\
Enhanced sensitivity to cytokines (e.g., TNF) & Adenovirus \\
IV. Sensitization to chemotherapy & Adenovirus \\
V. Expression of exogenous therapeutic genes & Herpesvirus \\
& Adenovirus \\
& Vaccinia \\
\hline
\end{tabular}

AExamples of agents for which mechanism has been demonstrated. CTL, cytotoxic T-lymphocyte; TNF, tumor necrosis factor. 
arterial or intravenous administration, as well. These studies also indicate that this novel biotherapy can interact in a potentially synergistic fashion with standard chemotherapeutic agents (13); a phase III randomized trial of cisplatin plus 5-fluorouracil (5-FU) with or without $d l 1520$ is to be initiated in the first half of 2000. Cross-resistance between agents with such radically different mechanisms of action is unlikely, and combinations may be well-tolerated, particularly if the toxicity profiles of each agent are nonoverlapping. For example, dl1520 causes flulike symptoms, whereas 5FU suppresses hematopoiesis and causes mucositis.

\section{Future directions}

Table 1 displays a number of replication-selective microorganisms that have recently entered clinical trials, as well as others that will follow shortly. Second generation agents are being engineered for greater potency while maintaining safety. To this end, vectors may be armed with genes that are intended to enhance local or systemic tumor destruction. T. Hermiston (in this Perspective series) considers strategies for gene delivery by biotherapeutic agents, and C. Springer (this series) addresses the use of prodrug-activating enzyme systems that could confer a high degree of specificity to tumor cell killing. In addition, S. Agha Mohammadi and M. Lotze (this series) discusses the potential of regulatable promoters in controlling expression of some transgene products. Methods of improving the intratumoral spread or the systemic delivery of viruses to metastatic tumors are also being explored. Pharmacologic modulation of the patient's immune responses may eventually be used to improve treatment efficacy and/or safety. Finally, combinations with standard chemotherapy, radiation and even other replicationselective microorganisms will be tested. These agents have different attributes, mechanisms of action and spectra of activity (Table 2) and should be complementary when used sequentially or in combination.

A number of different host and tumor-related factors will dictate the safety and efficacy of these agents. Possible examples include factors such as physical barriers to spread of the agent within a tumor, tumor growth fraction and genetics, viral receptor expression on tumor and normal tissues and the immune status of the patient. Many of these factors are certain to differ greatly between murine tumor model systems and cancer patients. Given the limited ability of preclinical tumor model systems to accurately predict the efficacy and therapeutic index of replication-competent agents in patients, the timely translation of encouraging agents into welldesigned clinical trials with relevant biological endpoints is critical. Only then can the true therapeutic potential of these agents be realized.

1. Dock, G. 1904. Rabies virus vaccination in a patient with cervical carcinoma Amer. J. Med. Sci. 127:563.

2. Asada, T. 1974. Treatment of human cancer with mumps virus. Cancer. 34:1907-1928.

3. Smith, R., Huebner, R.J., Rowe, W.P., Schatten, W.E., and Thomas, L.B. 1956. Studies on the use of viruses in the treatment of carcinoma of the cervix. Cancer. 9:1211-1218.

4. Webb, H.E., and Smith, C.E. 1970. Viruses in the treatment of cancer. Lancet. 1:1206-1208.

5. Coley, W.B. 1893. The treatment of malignant tumors by repeated inoculations of erysipelas with a report of ten original cases. Am. J. Med. Sci. 105:487-511.

6. Coley, W.B. 1911. A report of recent cases of inoperable sarcoma successfully treated with mixed toxins of erysipelas and Bacillus prodigiosus. Surg. Gynecol. Obstet. 13:174.

7. Morales, A., Eidinger, D., and Bruce, A. 1976. Intracavitary BCG in the treatment of superficial bladder tumors. J. Urol. 116:180-186

8. Mineta, T., Rabkin, S.D., Yazaki, T., Hunter, W.D., and Martuza, R.L. 1995. Attenuated multi-mutated herpes simplex virus-1 for the treatment of malignant gliomas. Nat. Med. 1:938-943.

9. Heise, C., et al. 1997. ONYX-015, an E1B gene-attenuated adenovirus, causes tumor-specific cytolysis and antitumoral efficacy that can be augmented by standard chemotherapeutic agents. Nat. Med. 3:639-645.

10. Lattime, E.C., Lee, S.S., Eisenlohr, L.C., and Mastrangelo, M.J. 1996. In situ cytokine gene transfection using vaccinia virus vectors. Semin. Oncol. 23:88-100.

11. Pawelek, J., Low, K., and Bermudes, D. 1997. Tumor-targeted Salmonella as a novel anti-cancer vector. Cancer Res. 57:4537-4544.

12. Kirn, D., et al. 1998. A phase II trial of intratumoral injection with an E1B-deleted adenovirus, ONYX-015, in patients with recurrent, refractory head and neck cancer. Proc. Am. Assoc. Clin. Oncol. 17:391a. (Abstr.)

13. Kirn, D.H., et al. 1999. A phase II trial of ONYX-015, a selectively-replicating adenovirus, in combination with cisplatin and 5 -fluorouracil in patients with recurrent head and neck cancer. Proc. Am. Assoc. Clin. Oncol. 18:1505. 